\title{
CAA modeling of helicopter main rotor in hover
}

\author{
Alexander N. Kusyumov ${ }^{1, a}$, Sergey A. Mikhailov ${ }^{2}$, Andrey S. Batrakov ${ }^{3}$, Sergey A. Kusyumov and George Barakos ${ }^{5}$ \\ ${ }^{1-4}$ Kazan National Research Technical University n.a. A. N. Tupolev (KNRTU-KAI), Kazan, Russia \\ ${ }^{5}$ University of Glasgow, Glasgow, UK
}

\begin{abstract}
In this work rotor aeroacoustics in hover is considered. Farfield observers are used and the nearfield flow parameters are obtained using the in house HMB and commercial Fluent CFD codes (identical hexa-grids are used for both solvers). Farfield noise at a remote observer position is calculated at post processing stage using FW-H solver implemented in Fluent and HMB. The main rotor of the UH-1H helicopter is considered as a test case for comparison to experimental data. The sound pressure level is estimated for different rotor blade collectives and observation angles.
\end{abstract}

\section{Introduction}

Main and tail rotors of conventional helicopters contribute to the generation of near-field and far-field noise [1]. Engine and fuselage noise are typically of secondary significance.

A helicopter main rotor generates primarily tonal and broadband noise. Tonal noise can be described as sum of two components: thickness and loading noise described by linear aerodynamic theory. The reason of thickness noise is the displacement of the fluid by the rotor blade and has a strong in-plane (rotor disk plane) contribution directed towards the flight direction. The term "loading noise" is usually a reference to the harmonic noise from non-impulsive loading sources. Loading noise is caused by the accelerating force generated by the moving blade surface. Basic loading noise during hover is generally dominant in a conical region directed 30 to 40 degrees downward from the rotor plane [2]. Thickness noise and loading noise are described by linear aerodynamic theory based on the Ffowcs Williams - Hawkings (FW-H) equation [2 - 4].

Additional sources, include Blade Vortex Interaction (BVI) noise and High Speed Impulsive (HSI) noise, and are dominant at specific flight regimes.

HSI noise is a particularly intense and is generated by helicopter rotors in high-speed forward flight. This HSI noise is closely associated with the appearance of shocks and transonic flow around the advancing rotor blades. Including the quadrupole sources in the FW-H equation [2] leads to account of nonlinearities in vicinity of the rotor blade. According to estimations (see, for example,
[5]) omission of the quadrupole sources in the FW-H equation leads to inaccurate results for tip Mach numbers higher than 0.7 .

Prediction of noise generation and propagation for aircraft design and certification falls in the area of Computational Aeroacoustics (CAA). CAA aims to combine flow data obtained by Computational Fluid Dynamics (CFD) for a nearfield area within a region near the aircraft surface. The sound perceived by a farfield observer is determined solving the wave propagation equation.

In the present work a task of design and validation of an in-house program on numerical solution of the FW-H equation is considered for a main rotor hover mode (unlike paper [6] that is based on a semi-analytical approach). The main rotor of $\mathrm{UH}-1 \mathrm{H}$ helicopter is considered as a test case for hover mode. Sound pressure level is estimated for different observation angles.

CFD data of the near field flow are obtained using the in-house HMB (University of Glasgow) code. In addition, results with the commercial ANSYS Fluent 13.0 code are obtained and the two data sets are compared. Farfield (thickness and load) noise obtained with an inhouse FW-H solver (compatible with the HMB code) are compared to experimental data [7] for a remote observer position located at the rotor disc plane. For arbitrary observation angle the results of the in-house $\mathrm{FW}-\mathrm{H}$ solver are compared to the data obtained with the $\mathrm{FW}-\mathrm{H}$ method in Fluent.

\footnotetext{
${ }^{1 \mathrm{a}}$ Prof., department of aero-hydrodynamics, postbox $7 @$ mail.ru

${ }^{2}$ Prof., department of aero-hydrodynamics, sergey.mikhaylov@kai.ru

${ }^{3} \mathrm{PhD}$ student, batrakov_a.c@mail.ru

${ }^{4}$ Teacher, department of aero-hydrodynamics, kusok88@yandex.ru

${ }^{5}$ Prof., School of Engineering, George.Barakos@glasgow.ac.uk
} 


\section{CFD Modelling}

The geometry of the rotor is presented in table 1 . Since temporal and spatial periodicity is assumed for the flow, the computational grid was constructed for a single rotor blade (with appropriate periodic boundary conditions). For the two-bladed rotor at hand, the computational domain is a half of a cylinder. The multi-block topology used in this paper, can be seen in Figure 1. C-type blocking is used around the blade. The structured and multi-block computational grid consisted of 88 blocks and 8.4 million cells. Along the aerofoil surface 218 points are located with concentration near the leading and trailing edges. Normal to the surface the first cell size is $10^{-5}$ of the blade's chord length and the cell ratio is less than 1.2 .

Table 1. UH-1H operational characteristics

\begin{tabular}{|l|c|l|}
\hline \multicolumn{1}{|c|}{ Parameter } & Value & \\
\hline Number of blades $N$ & 2 & \\
\hline Rotor radius $2 R, \mathrm{~m}$ & 14.63 & \\
\hline Rotor solidity $\sigma$ & 0.0464 & \\
\hline Blade chord $c, \mathrm{~m}$ & 0.53 & \\
\hline Blade airfoil & NACA 0012 & \\
\hline $\begin{array}{l}\text { Blade twist (root to } \\
\text { tip), deg }\end{array}$ & -10.9 & \\
\hline $\begin{array}{l}\text { Max gross weight } W, \\
N\end{array}$ & 43000 & \\
\hline
\end{tabular}

The multi-block topology used in this paper, can be seen in Figure 1.

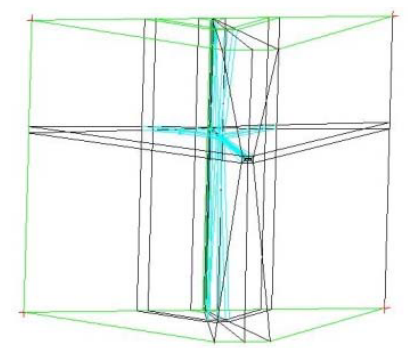

(a)

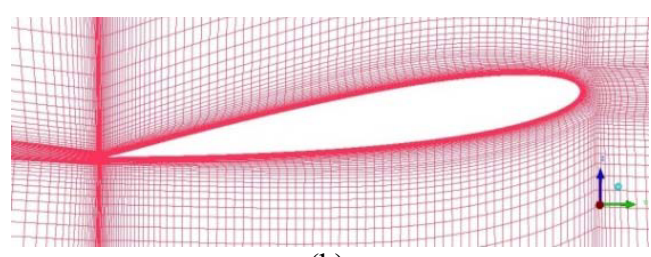

(b)

Fig 1. Multi-block topology (a) and mesh section (b)

The flow fields around the $\mathrm{UH}-1 \mathrm{H}$ helicopter rotor in hover were numerically simulated by solving 3D steady Reynolds-Averaged Navier-Stokes (RANS) equations.

Similar boundary conditions with some discrepancy at the bottom part of a modeling domain were used for the HMB and Fluent solvers.

For HMB the "source-sink" boundary conditions [8] were used at all surfaces of the computational domain apart from the symmetry plane. The "periodic" boundary condition provides the periodicity of the flow around the blade. Using the "inflow-outflow" boundary conditions allows for a reduction of the computational domain size. The inflow $\left(W_{\text {in }}\right)$ and outflow $\left(W_{\text {out }}\right)$ velocities were obtained from momentum theory according to the expressions:

$$
W_{\text {in }}=-\frac{U}{8} \sqrt{C_{T}}\left(\frac{R}{d}\right)^{2} ; \quad W_{o u t}=-U \sqrt{C_{T}} .
$$

Here $U$ is the speed of the blade tip; $C_{T}$ is the thrust coefficient; $d=2.5 R$ is the distance between the rotor centre and the top surface of the computational domain. The thrust coefficient $\left(C_{T}\right)$ is determined by the expression:

$$
C_{T}=\frac{T}{q_{\text {tip }} \pi R^{2}},
$$

where $q_{\text {tip }}$ is the dynamic pressure at the blade tip and $T$ is the rotor thrust, obtained after computation in iteration process. The rotor thrust coefficient $C_{T W}$ (under the condition $T=W$ ) is first used as an initial guess and then the thrust coefficient value $C_{T}$ is recomputed using successive approximations.

For the bottom part of computational domain the «pressure-outlet» boundary condition was assigned.

Simulations were conducted with the $k-\omega$ SST turbulence model. The flow conditions, and the values of the trust coefficient obtained with HMB and Fluent are presented in Table 2 . The collective pitch angle $\theta_{0.75}$ at the distance $r=0.75 R$ was choosen according to an approximate trimming method [9]. The values $W_{\text {in }}$ used for computations in both HMB and Fluent are shown in table 2. Some discrepancy between HMB and Fluent results (including difference between $W_{i n}$ ) can be explained, by the different type of boundary conditions at the bottom part of the computational domain.

Table 2. Conditions for computations

\begin{tabular}{|l|l|}
\hline Parameter & Value \\
\hline Collective pitch, $\theta_{0.75}$ & $3.3^{\circ}$ \\
\hline Tip Mach number, $M_{\text {tip }}$ & 0.8 \\
\hline Tip Reynolds number, Re & $9.5 \times 10^{6}$ \\
\hline Thrust coefficient (for $T=W$ ), $C_{T W}$ & 0.0055 \\
\hline Inflow velocity (for $T=W$ ), $W_{\text {in }}$ & $0.403 \mathrm{~m} / \mathrm{s}$ \\
\hline Inflow velocity (HMB), $W_{\text {in }}$ & $0.421 \mathrm{~m} / \mathrm{s}$ \\
\hline Inflow velocity (Fluent), $W_{\text {in }}$ & $0.2 \mathrm{~m} / \mathrm{s}$ \\
\hline Thrust coefficient (HMB), $C_{T}$ & 0.00577 \\
\hline Thrust coefficient (Fluent), $C_{T}$ & 0.0054 \\
\hline
\end{tabular}

Some distributed performances of flow around the rotor blades are presented in Figures 2 - 4. Figure 2 presents visualisation of the tip vortices structure using $Q$ criterion iso-surface based on the HMB code results. 


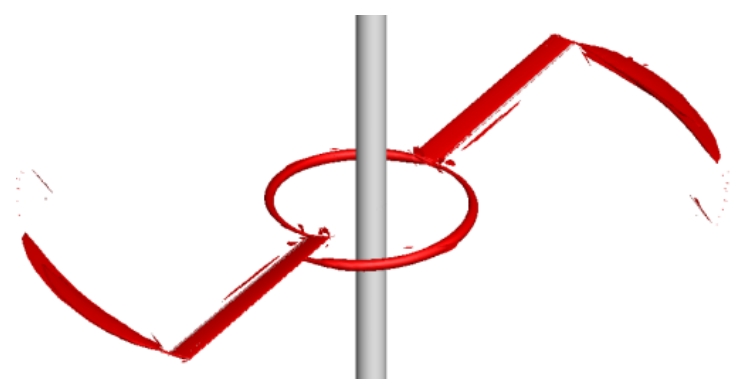

Fig 2. Tip vortex structure according to $\mathrm{Q}$-criterion $(\mathrm{Q}=0.01)$

Figure 3 presents results for the pressure coefficient distribution at different sections of the blade (normalized with the rotor radius, values of cross-sectional radius $\bar{r}$ ). Figure $3 \mathrm{a}$ shows the surface pressure coefficient distribution and location of the blade sections at $\bar{r}=0.75$, $\bar{r}=0.85, \bar{r}=0.95$.

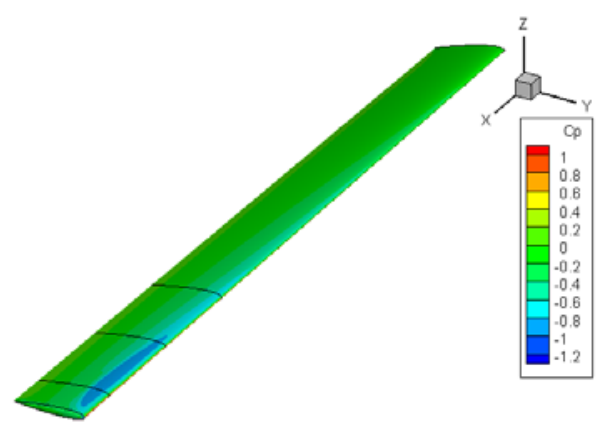

(a)

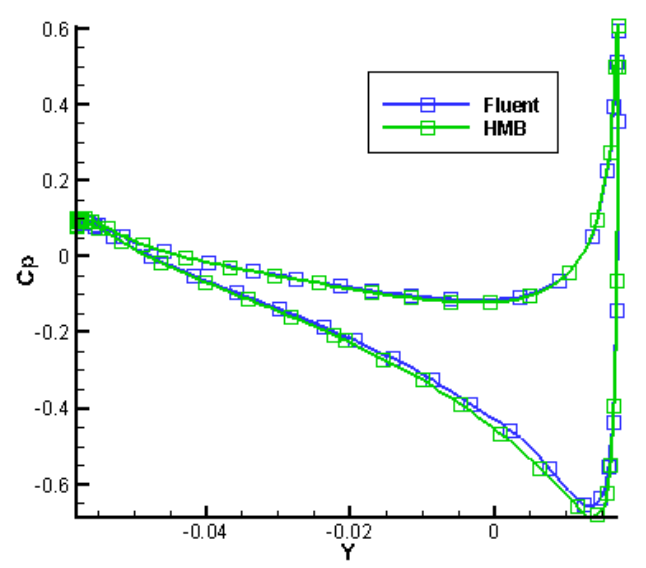

(b)

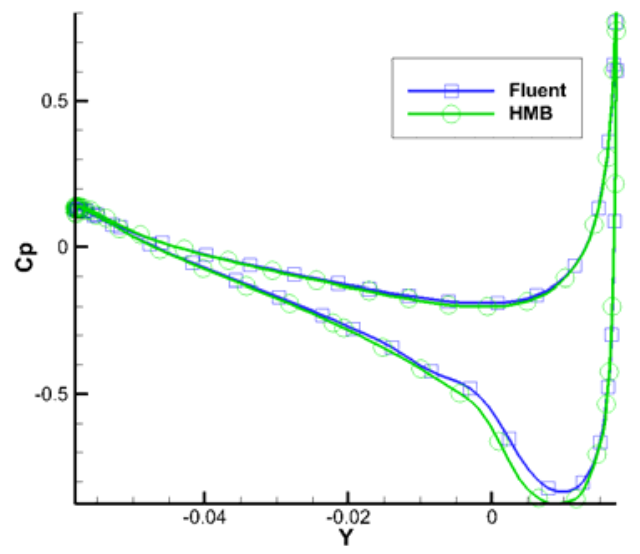

(c)

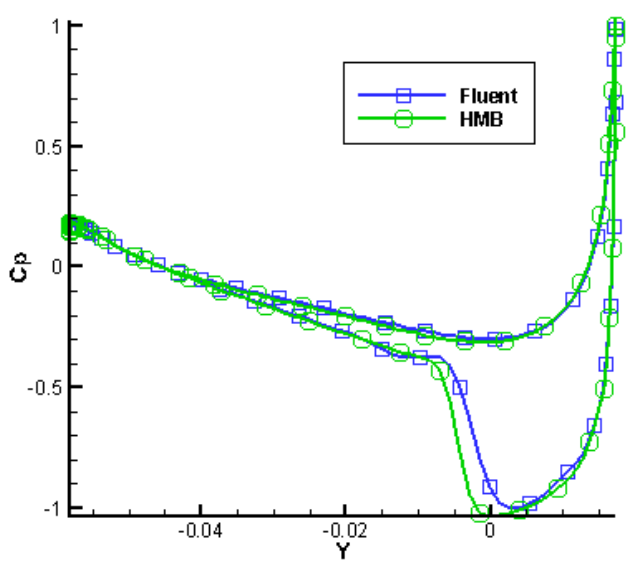

(d)

Fig. 3. Distribution of the pressure coefficient $C p$ on the UH$1 \mathrm{H}$ rotor blade surface (a) and at the blade sections: (b) $\bar{r}=$ 0.75 ; (c) $\bar{r}=0.85$; (d) $\bar{r}=0.95$.

Discrepancies between the pressure coefficient distributions obtained by HMB and Fluent lead to differences between the predicted values of the thrust coefficient (table 2).

The pressure coefficient $C p$ distribution (Figure 3) allows analysis of compressibility effects. From the comparison of $C p$ distribution on the top blade surface $(C p<0)$ a weak influence of compressibility effect is seen at sections $\bar{r}=0.75, \bar{r}=0.85$ (quite smooth $C p$ distribution). Stronger influence is seen at the section $\bar{r}=0.95$. This conclusion followed from the comparison of the local Mach number values distributions (HMB code results, Tecplot visualization) at the same sections in Figure 4: the local Mach number values $M \leq 0.4$ for $\bar{r}=0.75, \bar{r}=$ 0.85 and $M>0.4$ for $\bar{r}=0.95$.

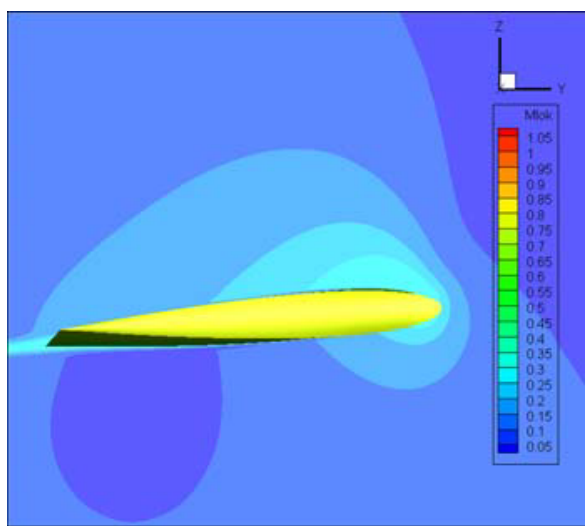

(a) 


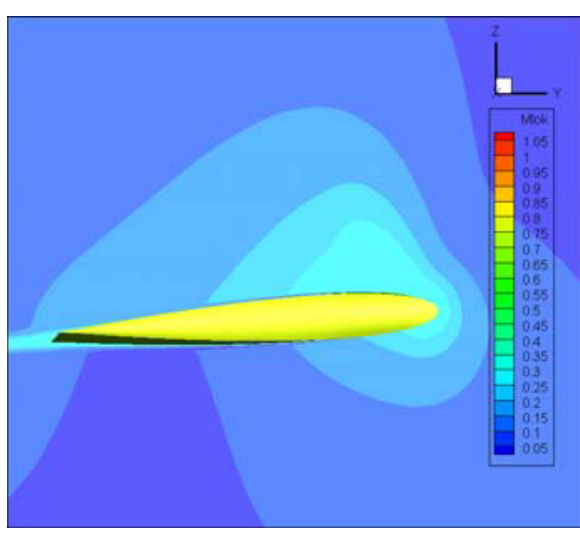

(b)

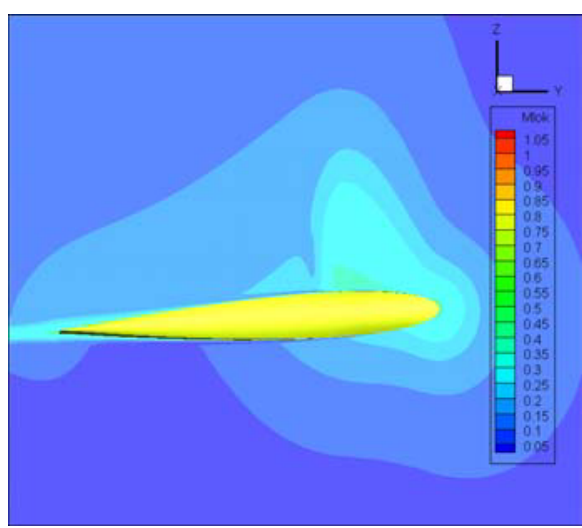

(c)

Fig. 4. Local Mach number distributions at the UH-1H blade sections: (a) $\bar{r}=0.75$; (b) $\bar{r}=0.85$; (c) $\bar{r}=0.95$.

From Figures 3 and 4 it follows that for the considered flow conditions around the UH-1H rotor blades the compressibility effect has significant influence on the flow parameters (and on the aeroacoustic emission power) close to the tip of blade $(\bar{r}>0.85)$ only. The geometry of the rotor blade, the surface pressure distribution and the condition of the flow are used as initial data for integration of the sound propagation FW$\mathrm{H}$ equation.

\section{FW-H equations}

A widely used method for predicting the aerodynamically generated rotor noise is based on the Ffowcs Williams Hawkings equation [3]. Implementation of the FW-H equation to CFD methods is usually based on integral formulation of the $\mathrm{FW}-\mathrm{H}$ equation. The $\mathrm{FW}-\mathrm{H}$ equation integral formulation includes several terms, including the thickness, load and quadrupole terms. In the present work the linear formulation of task is considered (quadrupole term, corresponding to the HIS noise is neglected).

The far field retarded-time formulation by Farassat [2] for the sound pressure is commonly referred as:

$$
4 \pi p^{\prime}(\mathbf{x}, t)=\frac{1}{a_{0}} \frac{\partial}{\partial t} \int_{f=0}\left[\frac{\rho_{0} a_{0} v_{n}+l_{r}}{r\left|1-M_{r}\right|}\right]_{r e t} d S .
$$

Here $a_{0}$ is sound speed; $f=0$ is a function that describes the blade surface; $\rho_{0}$ is the density of medium; $v_{n}$ is the local normal velocity of source surface; $M_{r}=M_{i} \hat{r}_{i}$ is the source Mach number in radiation direction; $r$ is the distance between observer and source, $r=|\mathbf{x}-\mathbf{y}|, \mathbf{x}$ is the observer position vector, with components $x_{i}, \mathbf{y}$ is the source position vector, with components $y_{i} ; p^{\prime}=p-p_{0}$ is the acoustic pressure, $p_{0}$ is the pressure of the medium, $p$ is the surface pressure; $l_{i}$ are the components of the local pressure force intensity, $l_{r}=l_{i} \hat{r}_{i}$. The subscript ret denotes the retarded time and the integration is performed over the actual blade surface $f=0$. For a moving surface the retarded time $\tau$ is determined by the expression

$$
|\mathbf{x}-\mathbf{y}(\eta, \tau)|=a_{0}(t-\tau),
$$

where $t$ and $\tau$ are the observer and the source time, and $\eta$ is the Lagrangian variable of a moving surface point.

Following the Farassat 1A formulation, the expression (1) can be rewritten in the form [2]:

Here

$$
p^{\prime}(\mathbf{x}, t)=p_{T}^{\prime}(\mathbf{x} ; t)+p_{L}^{\prime}(\mathbf{x} ; t) \text {. }
$$

$$
p_{T}^{\prime}(\mathrm{x} ; t)=\frac{1}{4 \pi} \int_{f=0}\left[\frac{\rho_{0} v_{n}}{r\left|1-M_{r}\right|^{2}}+\frac{\rho_{0} v_{n} \dot{M}_{r}}{r\left|1-M_{r}\right|^{3}}\right]_{r e t} d S
$$

is the thickness noise, and

$$
p_{L}^{\prime}(\mathbf{x} ; t)=\frac{1}{4 \pi a_{0}} \int_{f=0}\left[\frac{p \cos (\theta)}{r\left|1-M_{r}\right|^{2}}+\frac{p \cos (\theta) \dot{M}_{r}}{r\left|1-M_{r}\right|^{3}}\right]_{r e t} d S
$$

is the load noise. In these equations $\cos (\theta)=\boldsymbol{n} \cdot \hat{\boldsymbol{r}}(\boldsymbol{n}$ is a normal to emission surface), a dot over a variable implies the source time derivative.

As it was mentioned above the considered mathematical model of expressions (2) - (4), corresponds to the classical impermeable formulation of $\mathrm{FW}-\mathrm{H}$ equations: the source surface $f=0$ in this case corresponds to the blade surface. This mathematical model for the high blade tip Mach numbers usually gives under predicted values of the noise level. Nevertheless the classical FW-H formulation was used in the resent publications for relatively low blade tip Mach numbers values (see reference [10], for example). Then the acoustic pressure (based on the Formulation 1A) can be modified with the empirical adjustment, based on the radiation Mach number.

As the rotor blade behavior is characterized by complex motion in the computer program it is assumed that the blade is the rotating not deformed rigid body.

The employed algorithm is similar to the algorithm, described, for example, in [2]. The first stage of FWH solution algorithm is envisaged dividing the rotor blade surface into a number of panels. Integration over each panel is approximated using the value at the center.

Rotation matrices and an angular velocity vector are used to describe the blade rotation and the effects of pitch, cone and rotor disc tilt angles. Positions and velocities of points are determined in a blade-fixed stationary frame by combining the matrices/vectors with rigid body surface relations for rotating reference frames.

The summed effect of all the panels at the observer location gives the acoustic pressure time history. Described algorithm was implemented to the in-house HRS (Helicopter Rotor Sound) solver.

Figure 5 presents the results of numerical simulation for an observer located at the rotor disc plane on the distance of $3 R$ from rotor. From the comparison the total noise signatures predicted by the Fluent and HRS codes for an observer located in the rotor plane a good agreement is seen. 
The results of numerical simulation were compared also to the experimental data. Time observation shown in Figure 6 is $0.002 \mathrm{sec}$ for the rotation period of $0.0241 \mathrm{sec}$.

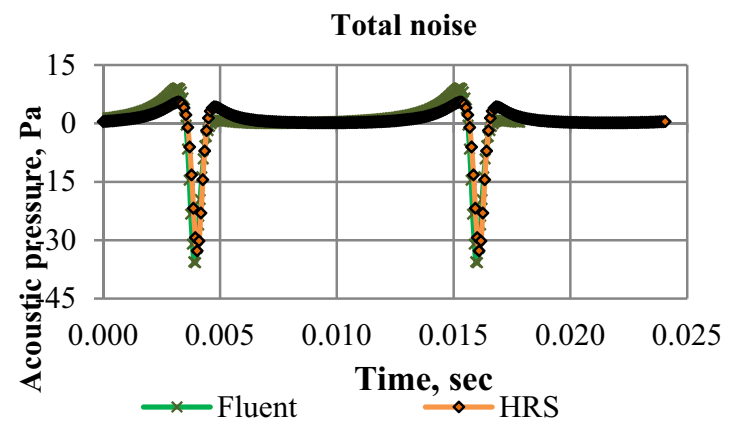

Fig. 5. Total noise predicted in the Fluent and HRS codes

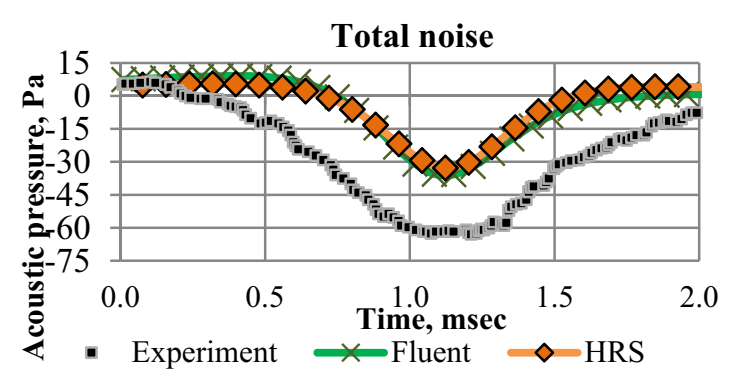

Fig. 6. Total noise predicted in the Fluent and HRS codes vs experimental data ([7])

Presented comparison of the Sound Pressure Level (SPL) predicted in the HRS solver corresponds to the SPL predicted in the Fluent code. It can be noted also a discrepancy between the experimental data and predicted by both (Fluent and HRS) solvers. This discrepancy is typical (see, for example, reference [2]) for accepted in the present work formulation of aeroacoustic task without taking into account the quadrupole term in $\mathrm{FW}-\mathrm{H}$ equation.

Figures 7, 8 present the total noise predicted in Fluent and HRS codes for the observers located out of the rotor plane. Reference [7] presents for hover mode results for the in-plane observer only. For arbitrary observation angle the HRS solver results are compared to the data obtained with the FW-H method in Fluent.

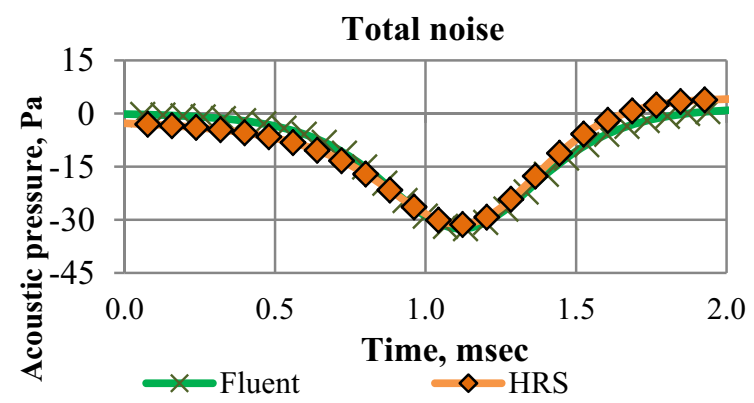

Fig. 7. Total noise predicted in the Fluent and HRS codes for the observation angle of 13 degrees

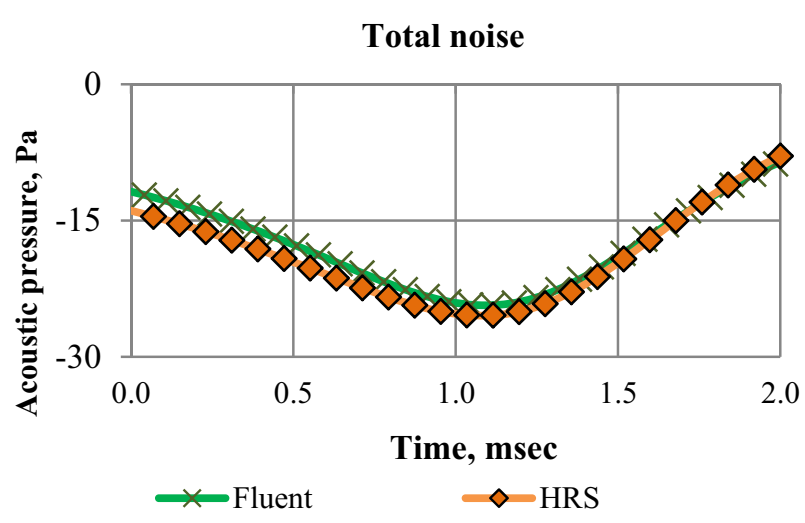

Fig. 8. Total noise predicted in Fluent and HRS codes for the observation angle of 26 degrees

It can be noted here that unlike the observation angle variation of the rotor blade collective angle for the values $\theta_{0.75}=0 ; 3.3$ and 6 degrees did not lead to strong change of the pick SPL values.

One can in general conclude that the results of numerical simulation of the UH-1H rotor in hover with HRS (HMB) codes are in good agreement to results of numerical simulation with the ANSYS Fluent code. Some discrepancy between the results of both solvers can be explained, in particular, by the presence of the discrepancy between predicted aerodynamical loads on the rotor blades.

\section{Conclusions and future work}

Validation of the in-house HRS code for the FW-H (Ffowcs Williams - Hawkings) numerical solution was considered. The total rotor noise was considered as the sum of the thickness and load noise based on linear formulation of aeroacoustics. To determine the nearfield flow parameters for the UH-1H rotor in hover mode, the HMB and Fluent CFD codes are used. The aeroacoustic FW-H option of the Fluent code was used also to determine the SPL (Sound Pressure Level) for comparison to HRS results at arbitrary observation angles. The results of aeroacoustic numerical simulation of the $\mathrm{UH}-1 \mathrm{H}$ rotor in hover with HRS (HMB) codes are in good agreement to the Fluent results. Discrepancy to the experimental data for the both solvers is explained by the linear formulation of the considered task (without the quadrupole term for the high speed noise).

In the future, the in-house HRS program for the rotor aeroacoustics will be revised for high blade tip Mach numbers.

Acknowledgement

This work is supported by the "Project part of state task in the field of the scientific activity" Grant (No 9.1694.2014/K). 


\section{References}

1. B. Edwards, Ch. Cox, NASA/CR-2002-211650 (May 2002).

2. K. S. Brentner, F. Farassat, Progress in Aerospace Sciences, 39 (2003).

3. J. E. Ffowcs Williams, D. L. Hawkings, Philosophical Transactions of the Royal Society, 264, no. 1151 (1969).

4. K. S. Brentner, NASA-TM-111251 Report, NASA Langley Research Center, Hampton, VA (1996).

5. A. S. Lyrintzis, Aeroacoustics, 2, no. 2 (2003).

6. A. Kusyumov, S. Mikhailov, L. Garipova, A. Batrakov and G. Barakos, International conference EFM-2015, Czech Republic (November 18-21, 2014).

7. F.H. Shmitz and Y.H., Yu, NASA Technical memorandum 84390 (1983).

8. N. A. Mohd, G. N. Barakos, RAeS Aerodynamics Conference, University of Bristol, UK (July 27-28, 2010).

9. J. Seddon, Basic helicopter aerodynamics, BSP professional books, 137 pp. (1990).

10. K. Sharma, K. S. Brentner, AHS 72nd Annual Forum, West Palm Beach, Florida, (May 17-19, 2016). 